\title{
Plague-Needs Continuous Surveillance System
}

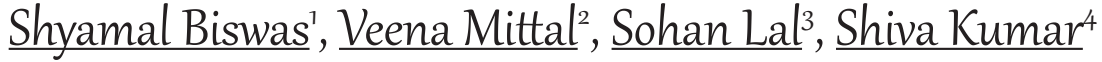 \\ ${ }^{1,3,4}$ National Centre for Disease Control, Plague Surveillance Unit, 8 Bellary Road, Bangalore, India. \\ ${ }^{2}$ National Centre for Disease Control, 22 Sham Nath Marg, Delhi, India. \\ DOI: https://doi.org/10.24321/0019.5138.202040
}

\section{I $\quad \mathbf{N} \quad \mathbf{F} \quad \mathbf{O}$}

\author{
Corresponding Author: \\ Shyamal Biswas, National Centre for Disease \\ Control, Plague Surveillance Unit, 8 Bellary Road, \\ Bangalore, India. \\ E-mail Id: \\ shyamalb07@gmail.com \\ Orcid Id: \\ https://orcid.org/0000-0002-7749-1018 \\ How to cite this article: \\ Biswas S, Mittal V, Lal S, Kumar S. Plague-Needs \\ Continuous Surveillance System. J Commun Dis \\ 2020; 52(4): 49-56. \\ Date of Submission: 2020-09-16 \\ Date of Acceptance: 2020-12-18
}

\section{$\begin{array}{llllllll}A & B & S & T & R & A & C & T\end{array}$}

Plague was considered to be a re-emerging disease since 1990s. The number of plague cases showed rising trend, and outbreaks were reappearing in various countries of the world after decades of quiescence. Plague is a zoonotic disease primarily of rodents. Natural decline in plague incidence would not justify the conclusion that plague has disappeared from the area Plague is transmitted between rodent and mammals via fleas. Rodents are the carriers of viral, rickettsial, nematode and bacterial diseases and are responsible for the transmission of more than 35 communicable diseases including Hanta viruses. Despite major advances in diagnosis, treatment, and prevention, it has not been possible to eradicate plague. The lack of continuous baseline surveillance resulted in an undetected sudden increase of the disease incidence in an enzootic/ endemic plague foci of the world, and a sudden outbreak of human cases, what was then considered a re-emerge of the disease. Regular plague surveillance work enhanced the possibility of detecting and delimiting plague foci and helped in determining the necessity for plague control programme. It is not uncommon to observe the long years of quiescence in natural plague foci and the sudden appearance of human cases is always destabilizing for national or even international authorities.

Keywords: Epidemic, Pandemics, Yersinia Pestia, Biovars, Resurgence, Enzootic, Sylvatic, Insecticide Resistant, Bamboo Flowering

\section{Introduction}

Plague is primarily a disease of sylvatic rodents, which are the inhabitants of natural foci where ecological conditions ensure the persistence of the plague organisms for a considerable period. Plague transmission occurs due to ecological imbalance when sylvatic rodents migrate from their natural habitats and intermingle with tolerant or susceptible rodent population in peri-domestic or domestic situations and transfer the infection through vector fleas causing cent percent mortality in susceptible rodent population (Rodent Epizootic or Rat fall). In absence of sufficient live rodents during rodent epizootics the infected fleas from the dead rodents attack/ bite the human population causing bubonic plague which later turns out as pneumonic form of plague. Plague, one of the most ancient, dreadful and formidable pestilential rodent borne zoonotic diseases continues to exist as a major public health problem in many countries of the world.

Scientists were in general opinion about the role of rodents and fleas in global transmission of plague and the main means of dissemination carried by infected rats through the international trade routes. Rodents are responsible for 
the transmission of more than 35 communicable diseases including plague, leptospirosis, typhus and Hanta viruses. In indirect transmission of diseases, rodents may serve as intermediate hosts for disease agents that ultimately infect man and may serve as reservoirs of the diseases which may be picked up by arthropod vectors like fleas, ticks or mites and transmitted to humans through bites. In direct transmission, rodents may transmit the disease agents by inhalation of aerosolized excreta, ingestion of excreta or by direct contact with the rodent itself and may directly transmit a pathogen to man through bite. ${ }^{1}$

A plague outbreak may cause widespread panic, as occurred in India in 1994 when a relatively small outbreak, with 54 deaths, reported in the city of Surat (Gujarat state) led to a nationwide collapse in tourism and trade.

Due to the high public health significance and the risk of its re-emergence after long years of quiescence the present paper is made to make us understand the importance of continuous surveillance for the forecasting of plague outbreaks and undertaking of suitable prevention and control measures. ${ }^{1,2,4,5,7,8}$

\section{Global Plague Situation}

According to WHO during the period 1987 to 2018, plague affected 28 countries with 57,946 cases and 4817 (8.31 percent) deaths. During the period the maximum number of plague cases (5419) occurred in 1997 and the minimum (243) occurred in 2018. Between 2004 to 2009, a total of 12,548 cases of human plague, including 845 (6.7 percent) deaths, were reported by 13 countries in Africa, Asia and Americas. The African countries (especially Democratic Republic of Congo and Madagascar) represented more than 97 percent of infections worldwide during 2000-2009. During 2013 to 2018, a total of 2886 cases and 504 (17.46 percent) deaths were recorded from 11 countries (Democratic Republic of Congo, Madagascar, Uganda, Tanzania, Bolivia, Peru, USA, China, Russian Federation, Kyrgyzstan and Mongolia) in the world. Despite the availability of a number of highly effective therapeutic agents, mortality due to plague in many countries was high during the period 1954-1997.

There were three periods of increased plague activity in the world over the past 44 years. The first one was during the mid-1960s, the second from 1973 to 1978 and the third was from the mid-1980s to the present particularly in Africa in the 1990s. Since the 1990s, plague was considered as a re-emerging disease, the number of plague cases showed rising trend, and outbreaks were reappearing in various countries of the world after decades of quiescence. Foci of plague have been detected in all continents, except Australia and Antarctica (Figure 1). 1-3,9,10 $^{-1}$

Red areas with potential plague natural foci based on historical data and current information (Source: WHO/ PED, as of 15 March 2016).

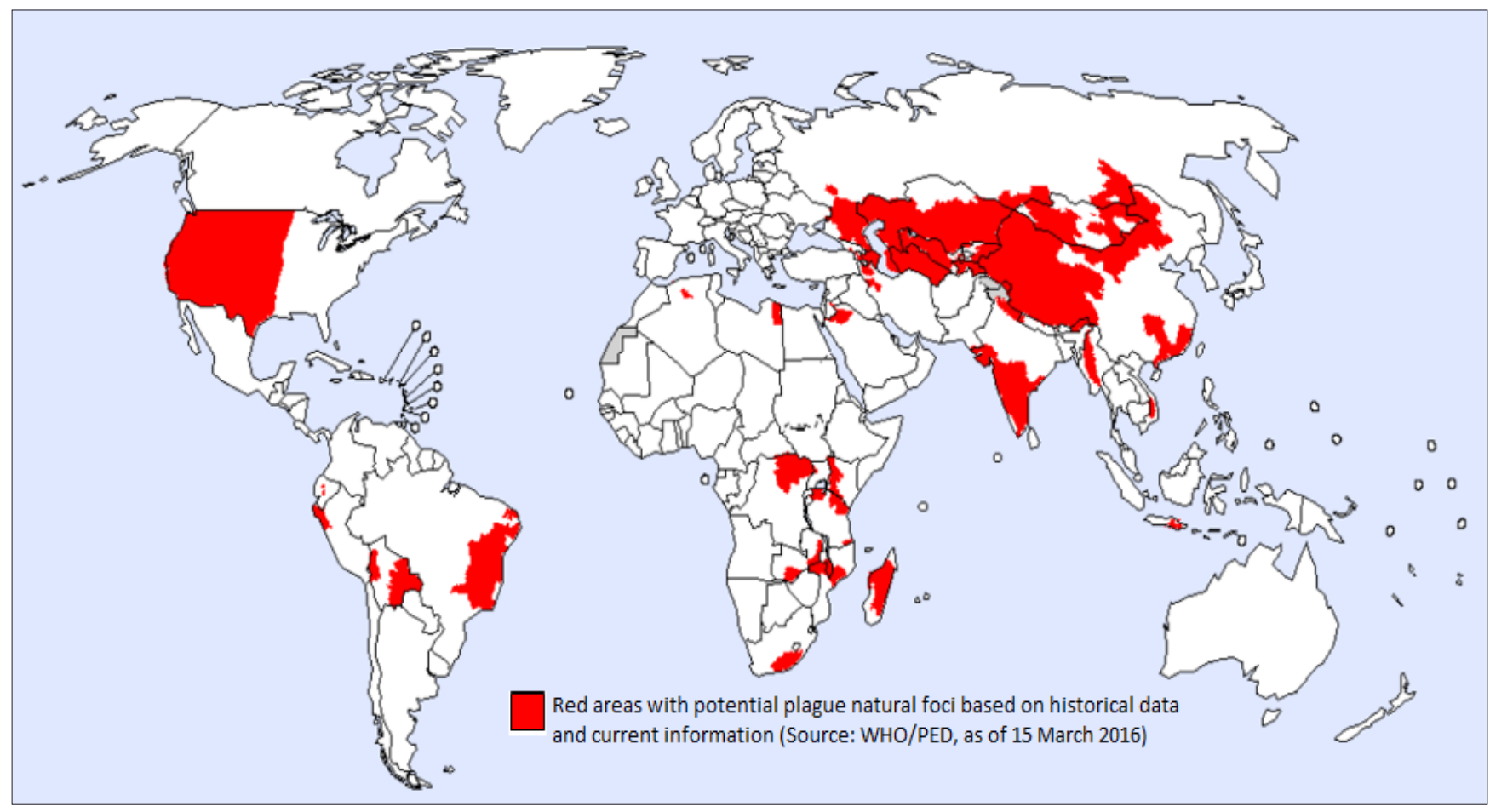

Figure I.Global distribution of natural plague foci as of March 2016 


\section{Plague in India}

Plague, was a major public health problem throughout India till the mid twentieth century A.D. The authenticated plague epidemic started in the year 1895-1896 and reached its peak in 1907. During the two decades from 1898-1928 there were over 12 million deaths in undivided India. The decennial death rate due to plague in India per 1, 00, 000 population during these decades were $183.3,133.8$ and 51.9 respectively. During 1949-1958 mortality rate due to plague was calculated to be 1.8 per $1,00,000$ population. There was a resurgence of plague in bordering districts of Tamil Nadu, Andhra Pradesh and Karnataka states during 1959 to 1966, since then mortality had declined and reached zero level during 1967. This may be attributed to discontinuation of DDT spray under National Malaria Eradication Programme in these areas. The last human plague case in India during the period was reported from Mulbagal in Kolar District, Karnataka in 1966. ${ }^{1,2}$

Sporadic cases of suspected human plague were reported from Tangnu, Himachal Pradesh during 1983, 1984 and Attibele, Karnataka in 1984. During 1994 a bubonic plague outbreak at Mamla village, Beed district, Maharashtra and pneumonic plague outbreak in Surat, Gujarat were recorded. Factors contributing to the re-emergence of plague in India in 1994, were ecological changes created by the earthquake in September, 1993 in Latur, Maharashtra that disturbed the equilibrium density of domestic rodents and their fleas in Beed district. Rainfall and flooding in Surat region, Gujarat that occurred before the outbreak, as well as Ganesh idol immersion that brought crowds of people together are also thought to have facilitated the spread of human plague (Table 1 ).

After 8 long years of quiescence a localized outbreak of pneumonic plague occurred in Hatkoti, Shimla district, Himachal Pradesh in 2002. In 2004, a bubonic plague outbreak occurred in Dangud village, Uttarkashi district, Uttarakhand. ${ }^{1,4,5,7,17,18}$

Table I.World Notification of Plague 1989 to 2009 after long years of quiescence ${ }^{\prime}$

\begin{tabular}{|c|c|c|}
\hline Country & $\begin{array}{c}\text { Year of } \\
\text { Reappearance }\end{array}$ & $\begin{array}{c}\text { Duration of } \\
\text { Quiescence }\end{array}$ \\
\hline Botswana & 1989 & 45 years \\
\hline Kenya & 1990 & 10 years \\
\hline India & 1994 & 27 years \\
\hline Indonesia & 1997 & 27 years \\
\hline Zambia & 1997 & 33 years \\
\hline Algeria & 2003 & 50 years \\
\hline Libya & 2009 & 25 years \\
\hline
\end{tabular}

\section{Objectives of Plague Surveillance}

- Exploration of the areas for future human cases and rodent epizootics,

- Identification of most common rodents and flea species maintaining enzootic foci of $Y$. pestis,

- Detection of sylvatic plague foci in the erstwhile plague endemic areas mainly to unravel the existence of sylvatic rodents in the maintenance of the reservoir of the infection,

- Undertaking the study on population stress under which migration takes place from sylvatic to peripheral and commensal habitats,

- Identification of host flea species as target for anti-flea measures,

- Collection of information on effectiveness of plague prevention and control measures,

- Identification of local ecological factors or human activities that result in increased exposure risks for humans,

- Study the trends in epidemiology and epizootology of plague in a given region.

\section{Passive Surveillance}

- To give advice on treatment and management of cases,

- To initiate efforts to identify the source of infection (Index case),

- To determine the extent of any rodent epizootic activity.

- To assess the potential for additional human cases,

- To disseminate the information on plague outbreak to health care personnel; and Implement emergency prevention (for bubonic plague transmission) and control measures (for pneumonic plague transmission),

- To collect the signs and symptoms of suspected plague cases for diagnostic confirmation by the trained doctors or plague surveillance workers,

- To implement emergency prevention and control measures.

\section{Active Surveillance}

- To identify the suspected case of human plague following rodent epizootics,

- To search the new cases by surveillance workers from clinical records in hospital and house to house search in the affected areas,

- To obtain serological test samples from the survivors and other members of the family and neighboring houses for plague antibody,

- To give prophylactic measures to the health workers in the affected areas as and when required,

- To collect the core information from each patient such as age; sex; occupation; residence including country, place of exposure if known or history of movement, source of exposure if known, date of onset, clinical 
presentation (bubonic, septicaemic, pneumonic), treatment taken, recovered or dead, possible exposure of others in contact with the patient, and preliminary classification of the case (suspected, presumptive or confirmed) following standard case definition,

- To collect all laboratory data including: types of samples analyzed (blood, sputum, bubo aspirate, serum, other), dates of sample collection, light and fluorescence microscopy results, chest $\mathrm{X}$-ray results, hematological findings, bacteriological results, results of serological tests and autopsy results for fatal cases,

- To collect the epidemiological investigation data for each human case to determine the source of infection and the risk of additional human cases. Reports of these investigations include: a complete history of the patients' activities and travel during the incubation period of the infection,

- $\quad$ To detect the source of infection (Rodents and fleas) and fever cases with lymphadenopathy,

- To estimate of number of people involved in plague transmission in the affected areas,

- $\quad$ To collect the data on possible exposure to $Y$. pestis infection in patient contacts (especially important for pneumonic plague cases).

\section{Human Surveillance}

- To collect blood sera and other appropriate samples from the suspected human plague cases to detect the fresh cases or the evidence of antibody against $Y$. pestis,

- To standardize the reporting system (Collection of basic information on clinical observations, treatment taken, laboratory test results and the data on epidemiology, rodent and vector surveillance),

- To collect the basic information on each suspected human plague case also include rodent activity and nuisance of fleas where the patients live, types of dwellings and storage of food in indoor/outdoor situations for the rodents,

- To collect and transport of samples (Blood, sputum, Bubo aspirate, serum etc.) in specified laboratory glass wares and onward transmission to the diagnostic centers immediately maintaining cold chain.

\section{Why Surveillance of Plague is Necessary?}

- Plague is a zoonotic disease primarily of rodents. Natural decline in plague incidence would not justify the conclusion that plague has disappeared from the area,

- With the decline in human plague incidence in a particular biotope the infection probably recedes to its original hosts, the wild rodents,

- Plague is not static but is shifted from place to place through contiguity of colony infection amongst wild rodents which eventually transferred the infection to the commensal rodents on their path,
- Many biotic and abiotic factors influence the persistence of the disease in wild population and causing epizootic plague,

- Can reappear after long years of quiescence,

- Rodent population cannot be eradicated. Rodent can adapt themselves in adverse condition to fill the ecospace.

\section{Surveillance of Plague in India}

Due to continuous surveillance from 1989 to 1994 serological evidence of plague was detected in wild and peri-domestic rodents in peninsular India. All the rodent species were shown to be the hosts for three important flea vectors i.e. Xenopsylla cheopis, Xenopsylla astia and Xenopsylla brasiliensis in the area. Plague is transmitted between rodent and mammals via fleas. Detection of plague foci was made in the erstwhile plague endemic areas mainly to unravel the existence of sylvatic rodents in the maintenance of the reservoir of the infection and to study the population stress under which migration takes place from sylvatic to peripheral and commensal habitats. As natural foci of plague are known to be completely independent of man, have a cyclic pattern of activity and can transmit the disease to man, a continuous surveillance and vigil has been maintained in India by National Centre for Disease Control (formerly N.I.C.D.), Ministry of Health, Govt. of India since $1963^{1,11}$ (Figure 2).

\section{Enzootic and Sylvatic Plague Foci in India}

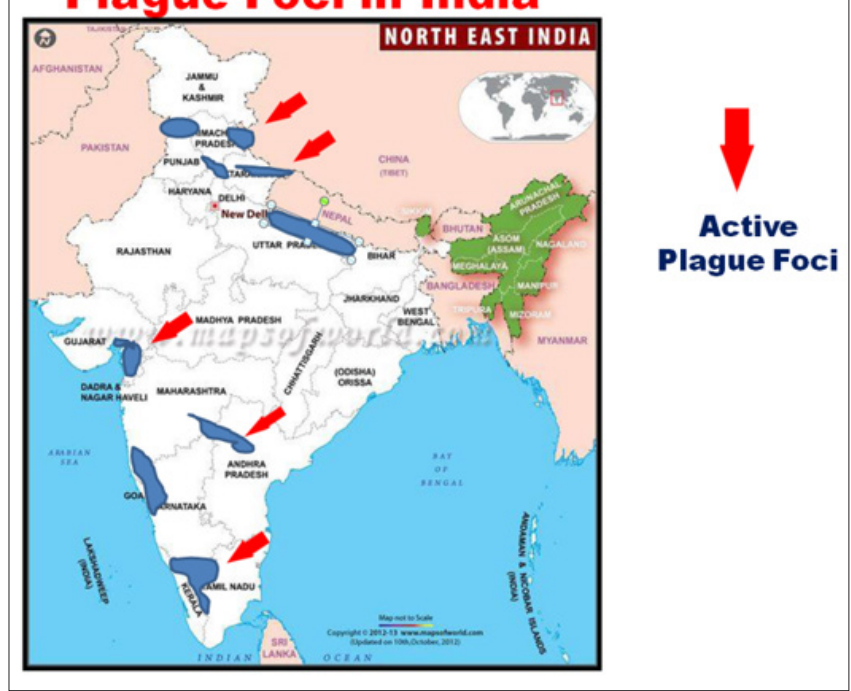

Figure 2.Enzootic and Sylvatic Plague Foci in India

\section{Surveillance Methodology}

\section{Surveillance of Plague Includes}

- Collection and estimation of rodent densities in a given biotope,

- Detection of plague antibodies among wild and commensal rodents, 
- Collection and identification of fleas and calculation of absolute and specific flea indice,

- Pathological examination of rodent organs,

- Examination of rodent organ smears for bi-polar bodies,

- Isolation of Yersinia pestis from rodent organ sample and fleas.

\section{Collection of Wild Rodents}

- Identification of live rodent burrows,

- Collection of wild rodents by digging (Figure 3),

- Collection of fleas from rodents,

- Transportation of rodents from the field to plague laboratory,

- Processing of rodents and fleas,

- Collection of blood and rodent organs,

- Preparation of rodent organ smears,

- Serological, bacteriological and entomological studies of the samples.
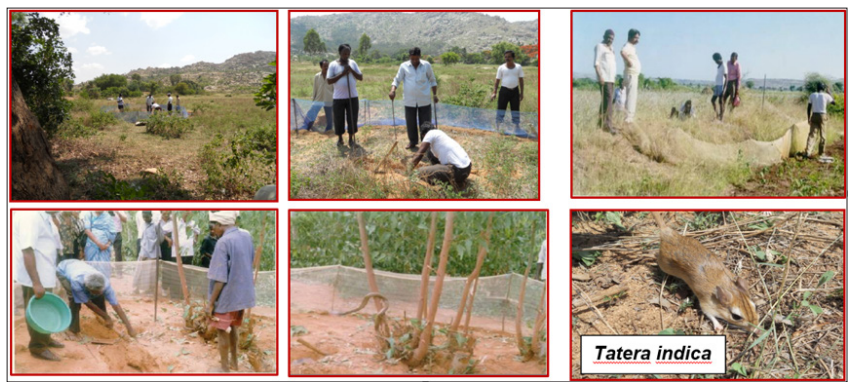

Figure 3. Collection of wild rodents from the fields by digging

\section{Collection of Domestic, Peri-domestic Rodents} and Fleas

- Collection of domestic and peri-domestic rodents by multiple catch live trap (Wonder trap) using appropriate baits (Dried fish, Bread butter, Peanut butter, Coffee powder, carrot pieces, ground nuts etc.) (Figure 4),

- Laying of traps during evening and retrieving of the traps next day in the morning,

- $\quad$ Transportation of traps with live rodents, covered with a cloth bag to avoid the free falling of rodent fleas from the live rodents (+ve or -ve for $Y$. pestis infection) in newer areas,

- Combing of trapped live rodents using an enamel basin to collect the live fleas and immature stages,

- Collection of live fleas from the basin using a suction tube ( $A$ hard glass test tube attached with two glass rods. One glass rod is attached with a small rubber bulb),

- Collection of live fleas for Identification up to species level, Insecticide susceptibility test, animal inoculation test and colonization in the laboratory.

\section{Processing of Rodents}

- Collection of blood samples from live rodents by puncturing heart using an injection syringe in a sterilized test tube or vaccutiner tube and collection of serum samples in the laboratory for serological and bacteriological investigation,

- Dissection of killed rodents for the collection of rodent organs from heart, lung, liver and spleen,

- Preparation of blood smears using portion of the dissected organs on a glass slide and detection of bipolar bodies under direct microscopy using Wayson stain,

- $\quad$ Storing of remaining organ portions in CBT medium for bacteriogical analysis.
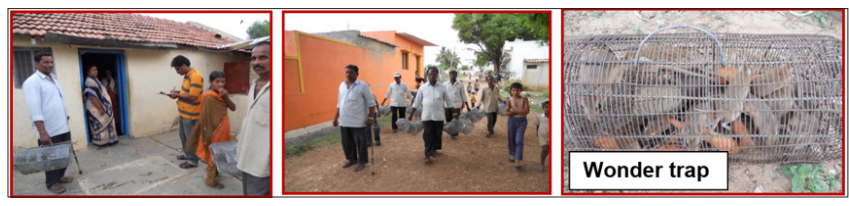

Figure 4.Collection of commensal and peri-domestic rodents

\section{Host Association of Fleas}

High flea indices cannot be co-related with rodent density in a given biotope. Fleas do not uniformly infest all rodents of a locality but show unequal distribution due to interference of certain natural ecological variation in the host-parasite relationship. Fleas usually spend part of their life as ectoparasite on rodent or other animals for feeding only. Adult fleas in large numbers are often found in loose soil in indoor situations and rodent burrows in peri-domestic and wild situations. Thus rat fleas retrieved only from the animal or rodent bodies may not give the actual flea index in a given area. ${ }^{1}$

\section{Carnivore Sero-Surveys}

- In plague endemic areas, dogs and cats in domestic and peri-domestic situations are exposed to $Y$. pestis and become infected via predation on infected animals or through flea bites. Plague infected dogs typically experience a milder form of the disease, presenting with nonspecific signs like lethargy, pyrexia and purulent skin lesions. Whereas, cats are highly susceptible to plague infection, presenting with a disease spectrum similar to that in humans. Mortality rate in untreated cats is equally high,

- Collection of blood/serum samples from carnivores to detect the evidence of plague activity, that consume rodent prey or likely to scavenge fresh rodent carcasses,

- Carnivore sero-surveys are mainly for the areas where vast areas to be sampled, no evidence of plague antibodies in local rodent populations, and rodent epizootics not occurred in surveyed areas for many years,

- Data can be obtained on sero-positive carnivores 
in plague-enzootic foci at any given time (A sudden increase in the percentage of sero-positive animals indicates that there is ongoing or recent epizootic activity in the area. Such a sudden rise in antibody serves as an early warning signal for the possible outbreak of plague in the area),

- Collection of blood samples from vein in the forelegs or hind legs from domestic or stray dogs that consume live rodents or fresh rodent carcasses. Dogs are to be properly restrained and muzzled (Figure 5),

- Pets such as dogs and cats may facilitate the transfer of infected fleas into homes through close living proximity and interaction with their owners.

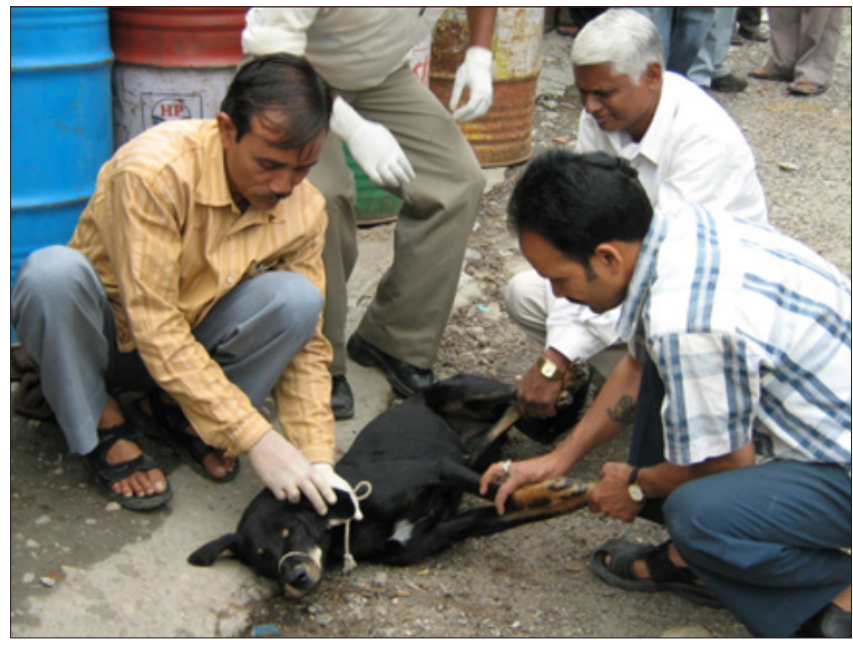

Figure 5.Collection of blood samples from stray dog

\section{Risk Factors in International Sea Ports}

The activities undertaken at ports, such as handling foodstuffs, attracts many species of vermin. Contaminated ships transport disease across geographical boundaries. Rodents can gain access to ships directly by mooring ropes, hulls and gang ways. Rodents may be concealed in cargo, ship's stores and other materials taken on to the ship ${ }^{1,12}$ (Figures 6 and 7).

\section{Mechanisms of Rodent Surveillance in Sea Port Areas}

- Surveillance in sea ports is carried out by the port health organization, a statutory body implementing the IHR2005 and the Indian Port Health Rules,

- Surveillance activities on board include cargo vessels, passenger vessels, sailing vessels and fishing vessels,

- Surveillance activities in and around port areas include the port installations and the residential colonies.

\section{Plague Surveillance Work at International Sea Ports}

- All the international air ports and sea ports in the country have to be kept free from rodents to prevent migration vis-à-vis transmission of rodent borne diseases particularly plague from endemic zones to disease free zones,

- $\quad$ Port Health Organizations issue the 'Deratting Certificates in accordance with Article 39 of the IHR-2005,

- Deratting means the procedure whereby health measures are taken to control or kill rodents in baggage, cargo, containers, conveyances, facilities, goods and postal parcels at the point of entry,

- Deratting certificates are valid for six months to national and international vessels on the basis of rodent free status of the ships,

- $\quad$ Port Health Organization inspects the proper use of 'Rat Guards' on anchored ropes and gang ways of the ships to prevent the entry of rodents to the main land or to the vessels. ${ }^{12}$

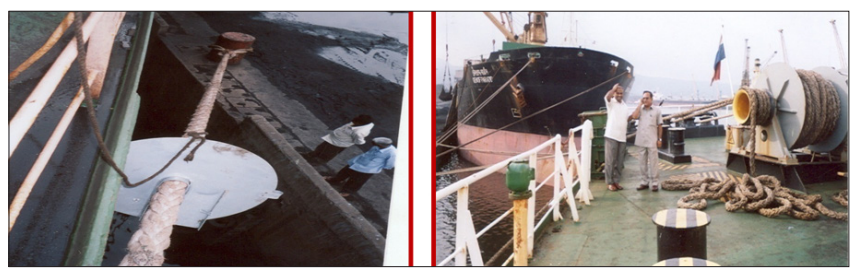

Figure 6.Use of rodent guard at mooring rope

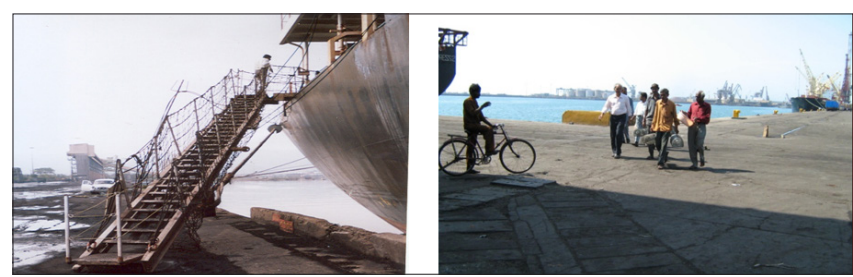

Figure 7.Entry and exit point for rodents (Gang way) collection of rodents in port areas

\section{Discussion}

Presently, plague continues to occur in Africa (particularly in Madagascar), the former Soviet Union, the Americas, and Asia (WHO Report). Under reporting of plague cases may be attributed to lack of diagnostic facilities for the confirmation of the cases and cessation of plague surveillance work by number of erstwhile plague endemic countries, where nonoccurrence of cases for long years gave false impression that Yersinia pestis are no longer circulating ${ }^{2,3,10}$

In Madagascar one case of Multi-Drug Resistance (MDR) in $Y$. pestis was reported in 1995 . The original isolate was resistant to chloramphenicol, streptomycin, sulfonamides, tracycline, and minocycline. Resistance to some of the typical alternative drugs such as ampicillin, kanamycin, and spectinomycin was also reported. Streptomycin, chloramphenicol and tetracycline are used for the treatment of plague, and sulfonamides and tetracycline used for prophylactic measures. The threat of antibiotic resistance in natural stains of $Y$. pestis may become an anti-measure problem since the resistance plasmid appeared to be freely transferrable between organisms. ${ }^{10}$ 
Multi-Drug Resistance (MDR) in $Y$. pestis and insecticide resistance in vector fleas to organochlorine, organophosphate and synthetic pyrethroids in most of the endemic countries may pose a serious threat to global outbreak of the disease. ${ }^{1,10}$ The resistance of fleas to various insecticides gave us warnings for appropriate use of residual insecticides and insecticide dust powders (insufflations) for efficient anti flea measures. Since newer pesticide molecules are being flooded into the market and the availability of recommended old pesticides becoming scarce, farmers unknowingly purchase and spray some non-recommended pesticides to solve the pest problem. The indiscriminate use of insecticides in agricultural sector has direct impact on resistance problem in flea population breeding outdoors. Effective flea resistance management depends on early detection of the problem and rapid assimilation of information on the resistant insect population so that rational pesticide choices can be made. Many insecticides are also massively used to control domestic pests, and therefore, impact will be more on the vector species which are breeding and resting indoors like fleas. Furthermore, in some circumstances, resistance can persist in populations for very long periods after regular use of an insecticide has ceased. In these cases, resistance to new insecticides is inherited from the past as a result of the previous use of other insecticides.

Deforestation had ultimate consequences in plague outbreaks as observed in Madagascar. First relates to the possibility of enhanced contact opportunities of humans with sylvatic plague in the wild situations; the second relates to the change in landscapes favoring rodents to develop as plague reservoirs. ${ }^{10}$

Compared to the previous 100 years, global changes in climate and severe weather conditions in recent decades are believed to have a growing and significant influence on human health. Climatic change has now been recognized as a contributing factor for the changes in ecology and epidemiology of infectious diseases including plague. The influence of climactic factors on the epidemiology of plague was noticed on or before 1960s. It was observed that the timing of outbreaks was strongly influenced by temperature and rainfall patterns. Global warming may accelerate plague spread in warm, tropical regions.

The inadequate management of the outbreaks in Beed and Surat (1994) triggered improvements in the surveillance, prevention and control of plague in India. As a result, the outbreak of pneumonic plague in Shimla (Himachal Pradesh) in 2002 and bubonic plague outbreak in Uttarkashi district, Uttarakhand in 2004 were contained in the shortest possible time. Early diagnosis, antibiotic treatment, mass chemoprophylaxis, quarantine and vector control measures were performed in timely manner. ${ }^{17,18}$

Rodent and vector surveillance is useful for detecting plague circulation in reservoir populations, assessing epidemic risk factors and surveying the susceptibility of fleas to insecticides. In addition to routine surveillance activities, new strategies based on use of geographical information systems, climatic and ecological data and mathematical models are improving the prediction of outbreaks.

International sea ports were the entry point for plague. Ships arrived from plague-endemic countries brought the infected rodents and fleas with $Y$. pestis infection. Potential new foci should be confirmed and investigated, with special attention to harbours with international trade. Although the number of human cases of plague is relatively low, it would be a mistake to overlook its threat to humanity, because of the disease's inherent communicability, rapid spread, capability of the agent to cause epidemic, rapid clinical course, high mortality if left untreated and international importance and economic impact. Yersinia pestis bacterium is widely available in microbiology banks around the world, making a biological attack a potential problem. ${ }^{1,12}$

Despite the advancements in diagnosis, treatment and advances in scientific knowledge and understanding of plague transmission, public perception and response to reemerging plague today is the similar that of in the past and historic times. Reemergence of plague of bubonic and/or pneumonic plague after 27 years of quiescence with only 54 deaths in Surat, Gujarat, India caused widespread panic. The news of outbreaks spread so quickly and resulted in a mass exodus of more than 500,000 people from the city of Surat and posed a threat of transmission to newer region. Many countries suspended air traffic and cargo movement from and to India. This led to a nationwide collapse in tourism and trade. The total economic loss due to the collapse of trade and tourism was estimated to be US\$600 million. . $^{1,10,13-16}$

Now, regular surveillance of plague is not being carried out in India due to non-availability of indigenous serological test kits (being manufactured by Haffkine Institute, Mumbai, India) and closing of most of the state plague surveillance units in the endemic states. ${ }^{1,17,18}$

Plague should not be relegated to the sidelines. It remains a poorly understood threat that we cannot afford to ignore. ${ }^{1}$

\section{Conclusion}

Plague is a re-emerging zoonotic disease. The number of human plague cases reported to W.H.O. by different countries was always lower than the actual number of cases. Under reporting may be attributed to lack of diagnostic facilities for the confirmation of the cases and cessation of plague surveillance work by number of erstwhile plague endemic countries. Under International Health Regulation (IHR 1969), plague was one of the International notifiable diseases. Countries were required to report all cases of human plague to $\mathrm{WHO}$, whereas, under revised regulations 
(IHR 2005) these requirements changed. Actual occurrence of plague cases and deaths cannot be assessed as the endemic countries may not report under Public Health Emergency of International Concern (PHEIC: IHR 2005). ${ }^{1}$

The worldwide decline in plague incidence since the 1950s resulted in decreased financial support, lesser interest, and ultimately the deterioration of laboratory-based surveillance systems in many endemic countries in the world. The lack of continuous baseline data on plague surveillance may result in an undetected sudden increase of bubonic/pneumonic cases in an enzootic/endemic foci of the world, or re-emerging of the disease. Plague can re-emerge, vaccination is useless and mass killing of rodents is not the solution for the eradication of the disease. According to the World Health Organization (WHO), global incidence of human plague was the lowest reported in 30 years, and the actual incidence was probably little different. However, we should be extremely vigilant as it is not uncommon to observe the long years of quiescence in natural plague foci, and the sudden appearance of human cases is always destabilizing for national or even international authorities. Despite major advances in diagnosis, treatment, and prevention, it has not been possible to eradicate plague.

Most of the State Plague Surveillance units in India stopped working due to non-recruitment of field staffs and availability of proper infrastructure. Due to discontinuation of regular plague surveillance work by the endemic states in India, national plague surveillance programme and regional collaboration are needed to be strengthened. Plague Surveillance Unit of NCDC, Bangalore and central Plague laboratory, NCDC, Delhi may be entrusted to undertake regular plague surveillance work in all the seven plague endemic states, erstwhile plague endemic areas and international sea ports in India.

\section{Conflict of Interest: None}

\section{References}

1. Biswas S. Plague in India: a review. India J Com Dis 2018: 50 (3): 60-75.

2. Plague around the world 2010-2015. WHO, Weekly Epidemiological Record. 2016; 91: 89-104.

3. Bertherata E. Plague around the world in 2019. WHO, Weekly Epidemiological Record, 2019.

4. Seal SC. Plague in India, reprinted from Proceedings of the National Institute of Science of India. 1960; 26B (Suppl.).

5. Plague Control in India, Directorate General of Health Services, Ministry of Health and Family Welfare, Government of India. PEEPEE Publishers and Distributors (P) Ltd., 2005.

6. Ramalingaswami V. Plague in India. Nat Med 1995; 1: 1237-9.

7. Datta KK. Plague: epidemiology, prevention \& control. National Institute of Communicable Diseases,
Directorate General of Health Services, Ministry of Health and Family Welfare, Government of India. 1994; $1-55$.

8. World Health Organization, Regional Office for South East Asia, Operational Guidelines on Plague Surveillance, Diagnosis, Prevention and Control. 2009.

9. Jae-Llane D, Hodgkins R. Yersinia pestis, a problem of the past and a re-emerging threat. Bio-safety and Health 2019; 1(2): 65-70.

10. Boire NA, Riedel VAA, Parrish NM et al. Lessons learned from historic plague epidemics: the relevance of an ancient disease in modern times. J Anc Dis Prev Rem 2014; 2 : 114.

11. Shyamal B, Lal S, Veena M. Detection of Enzootic Plague foci in Peninsular. India J Com Dis 2011; 43(3): 169-176.

12. Shyamal B, Nathan S, Mittal V et al. Surveillance of rodent and fleas and prevention of plague in international sea ports in India in the context of International Health Regulations-2005. Journal of Communicable Diseases 2015; 47(3): 33-41.

13. Shyamal B, Kumar RR, Kumar S et al. Susceptibility status of Rat Flea, Xenopsylla Cheopis (Siphonaptera: Pulicidae) vector of human plague against Organochlorine, organophosphate and synthetic Pyrethroids in Beed District, Maharashtra. India Journal of Communicable Diseases 2015; 47(3): 42-45.

14. Shyamal B, Kumar RR, Vaishnav KG et al. Study on the impact of insufflation and residual insecticide spray against Rat Fleas and present susceptibility status of Rodent Fleas to various insecticides in Surat, Gujarat. Journal of Communicable Diseases 2015; 47(3): 26-32.

15. Shyamal B, Ravi Kumar R, Sohan L et al. Present susceptibility status of rat flea Xenopsylla cheopis (Siphonaptera: Pulicidae), vector of plague against organochlorine, organophosphate and synthetic pyrethroids 1 . The Nilgiris District, Tamil Nadu, India. J Commun Dis 2008; 40(1): 41-45.

16. Biswas S, Ravi K, Shiv K et al. Sericulture and the development of resistance to various insecticides in Xenopsylla cheopis (Rodent flea), efficient vector of human plague in active enzootic plague foci of Kolar District, Karnataka, and Chittoor District, Andhra Pradesh, India. J Commun Dis 2016; 48(2): 36-41.

17. Mittal V, Bhattacharya D, Rana UV et al. Prompt laboratory diagnosis in timely containment of a plague outbreak in India. J Commun Dis Dec 2006; 38(4): 317 324.

18. Mittal V, Rana UV, Jain SK et al. Quick control of bubonic plague outbreak in Uttar Kashi, India. J Commun Dis Dec 2004; 36(4): 233-239. 